\title{
Granuloma eosinófilo craniano na infância
}

\author{
Carlos Umberto Pereira*, João Domingos Barbosa Carneiro Leão**, Alvino Dutra da Silva**, \\ Clarissa de Paula Rocha Santos ${ }^{* * *}$, Egmond Alves Silva Santos ${ }^{* * *}$ \\ Serviço de Neurocirurgia do Hospital João Alves Filho. Aracaju, SE
}

\section{RESUMO}

O granuloma eosinófilo é uma das três variantes de um grupo de doenças denominado histiocitose de células de Langerhans. O granuloma eosinófilo solitário do crânio tem sido considerado raro na infância. Durante o período de janeiro de 1997 e junho de 2003, foram estudados seis casos de granuloma eosinófilo craniano na infância. O sexo masculino foi o mais acometido (4:2). A idade variou dos 4 meses a 12 anos, com média de 5,5 anos. A localização craniana foi: parietal em três, temporal em dois e frontal em um. Os sintomas mais freqüentes foram tumefação craniana e dor local. Radiografia simples e tomografia computadorizada identificaram a lesão e orientaram na conduta. O tratamento cirúrgico foi instituído em todos os casos, com ressecção total da lesão. O seguimento desses pacientes foi possível em quatro que, até a presente data, encontram-se bem.

\section{PALAVRAS-CHAVE}

Histiocitose X. Granuloma eosinófilo craniano.

\section{ABSTRACT}

Eosinophilic granuloma of skull in infancy

Eosinophilic granuloma is one of the three Langerhan's cells histiocytosis. The skull eosinophilic granuloma has been considered rare in childhood.

From January, 1997 to June, 2003, six cases of eosinophilic granuloma of the skull in children were admitted. Males were more affected (4:2). The age ranged from four months to twelve-years (medium: 5.5 years). The localization of the lesion in the skull was parietal in three cases, temporal in two and frontal in one.

The most frequent symptoms were tumefaction and local pain. Skull X-ray and computed tomography were diagnostic.

Surgery for extirpation of the lesion was done in all cases. Follow-up of these patients was possible in four, with a good evolution up to now.

\section{KEYWORDS}

Histiocytosis X. Eosinophilic granuloma of skull.

\section{Introdução}

O granuloma eosinófilo (GE) é uma doença descrita como a forma mais benigna das histiocitose X. É caracterizada pela proliferação anormal de histiócitos, localizada ou multifocal, acometendo, principalmente, os ossos do crânio, costelas, pelve mandíbula, fêmur e coluna vertebral, sendo mais freqüentes em crianças e adolescentes ${ }^{15}$.

Os possíveis fatores envolvidos nessa doença são: resposta inflamatória a um agente etiológico não identificado, disfunção do sistema imunológico e alterações metabólicas; porém, até o presente, não existe comprovação definitiva $a^{10,17,30,31}$. Sabe-se apenas que a doença não é hereditária ou familiar, não é contagiosa e não apresenta predomínio racial ${ }^{13}$.

Os autores descrevem seis casos de GE no crânio em crianças e discutem sua localização, diagnóstico, tratamento e prognóstico.

\section{Casuística}

Durante o período compreendido entre janeiro de 1997 e junho de 2003, foram internados no Serviço de

\footnotetext{
*Professor Adjunto Doutor do Departamento de Medicina da Universidade Federal de Sergipe e Neurocirurgião do Hospital João Alves Filho. Aracaju, SE.

**Neurocirurgiões do Serviço de Neurocirurgia do Hospital João Alves Filho. Aracaju, SE.

****Médica do Hospital João Alves Filho. Aracaju, SE.

****Doutorando de Medicina da Universidade Federal de Sergipe. Aracaju, SE.
} 
Neurocirurgia do Hospital João Alves Filho (Aracaju, $\mathrm{SE}$ ), seis pacientes portadores de granuloma eosinófilo solitário craniano. A idade variou entre 4 meses e 12 anos, com uma média de 5,5 anos. Houve predomínio do sexo masculino (4:2). A localização craniana foi: parietal em três casos, temporal em dois e frontal em um caso. Os sintomas mais freqüentes foram tumefação craniana e dor local. Craniograma simples identificou a lesão osteolítica em todos os casos (Figura 1). Tomografia computadorizada realizada em todos os casos demonstrou a lesão e o envolvimento das estruturas adjacentes em todos os casos e foi importante na orientação do tratamento cirúrgico (Figura 2).



Figura 1 - Radiografia simples de crânio em perfil demonstrando lesão osteolítica na região parietal.

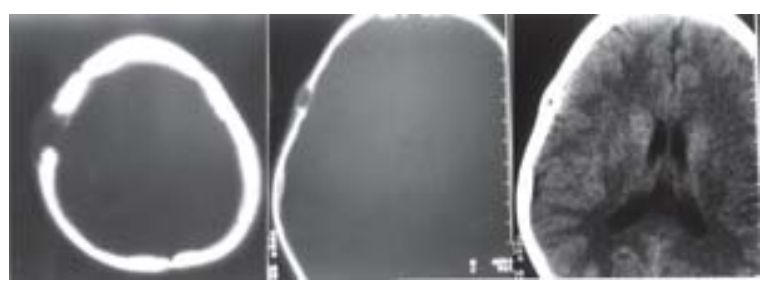

Figura 2 - Variadas aparências do granuloma eosinófilo na tomografia.

O tratamento cirúrgico foi instituído em todos os casos e consistiu na ressecção total da lesão. O acompanhamento foi possível em quatro desses pacientes e, até o momento, apresentam boa evolução.

\section{Discussão}

O termo histiocitose $\mathrm{X}$ foi introduzido por Lichtenstein em 1953, para denominar um grupo de doenças de etiologia desconhecida, caracterizado por proliferação anormal de histiócitos, que inclui a doença de LettererSiwe, doença de Hand-Schüller-Christian e o granuloma eosinófilo ${ }^{18}$. Esta classificação baseia-se no número de sistemas envolvidos na doença, idade do paciente e localização inicial do processo mórbido. Atualmente, o termo histiocitose de células de Langerhans (HCL), introduzido, em 1987, pela Histiocyte Society, é preferido pela maioria dos autores ${ }^{2,3,4,8,14,17,23}$.

O GE é uma doença descrita como sendo a forma mais benigna da histiocitose $\mathrm{X}$, caracterizada pela infiltração de histiócitos, eosinófilos, macrófagos e linfócitos $\mathrm{T}$ na cavidade medular do osso formando lesões osteolíticas, que pode ser localizada ou multifocal, afetando principalmente os ossos do crânio, costelas, mandíbula, fêmur e coluna vertebral, sendo mais freqüente em crianças e adolescentes ${ }^{15}$. O GE representa $50 \%$ a $60 \%$ dos casos de HCL e acomete pacientes entre 5 e 15 anos de idade, porém, pode acometer qualquer faixa etáriaa ${ }^{16,22,31}$. Em nossa casuística a idade variou de 4 meses a 12 anos, com uma média de 5,5 anos.

O crânio é o local mais afetado pelo GE ${ }^{26,31}$, sendo a abóbada craniana mais acometida que a base do crânio $^{22,31}$. O osso parietal é o mais afetado, seguido do frontal ${ }^{7,26}$. A literatura médica mostra um envolvimento do osso temporal entre $18 \%$ e $61 \%$ dos $\operatorname{casos}^{13,14,21,27}$. Lesões localizadas no parênquima cerebral são raras, e a maior parte destas resulta de lesões contíguas do crânio ${ }^{12,20,28}$. Quando ocorre envolvimento do sistema nervoso central, as áreas mais envolvidas são hipotálamo e hipófise, manifestando-se na maioria dos


pacientes, a localização parietal foi a mais comum, seguido da temporal e frontal, não havendo nenhum caso de acometimento do parênquima cerebral .

As queixas mais freqüentes do GE craniano são dor, presença de massa localizada com consistência macia e aumento da sensibilidade local ${ }^{1,5,7,9,17,19,26,29}$. As manifestações clínicas também estão relacionadas com a localização da lesão 5 . Os sintomas do GE craniano variam de acordo com a evolução do quadro. Inicialmente, quando o tumor está em desenvolvimento intradiplóico é assintomático. A dor é, geralmente, o primeiro sintoma, aparecendo à medida que o tumor destrói a tábua externa, díploe e tábua interna da calota craniana e, finalmente invasão da dura-máter 5 . Em nossos pacientes, a dor e tumefação localizada foram as queixas mais comuns, o

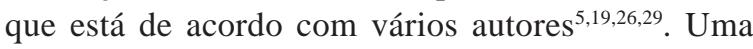
apresentação incomum e GE cranianao é a formação de hematoma epidural que pode estar relacionado com história de traumatismo craniano leve ${ }^{7}$.

Os exames laboratoriais podem demonstrar anemia leve, leucocitose, leucopenia, eosinofilia e velocidade de hemossedimentação elevada ${ }^{8}$. Pode haver elevação da 
fosfatase alcalina e das imunoglobulinas ${ }^{19}$. Os métodos de imagem são muito importantes para ajudar estabelecer o diagnóstico e tratamento. Na radiografia simples de crânio, o GE apresenta-se como uma lesão osteolítica, bem delimitada e com aspecto em "saca-bocado" "5,8,19,25,26,31 . A tomografia computadorizada (TC) demonstra a presença de uma lesão osteolítica, sua extensão e comprometimento intracraniano ${ }^{11,31}$. A TC em nossos pacientes demonstrou lesões osteolíticas em todos os casos; não havia invasão intracraniana em nenhum. A ressonância magnética define a extensão do envolvimento ósseo e dos tecidos moles adjacentes; as lesões aparecem com sinal hipointenso em T1, hiperintenso em T2 e há realce após administração do gadolínio ${ }^{2,6}$.

O diagnóstico diferencial deve ser feito com osteomielite, tuberculose, sífilis, cisto solitário do osso, tumor de células gigantes, mieloma múltiplo, metástases de neuroblastoma ou linfoma, osteíte fibrosa cística $^{8,11,12,19,29,30}$. Quando localizado no osso temporal, o diagnóstico diferencial inclui colesteatoma, mastoidite crônica, rabdomiossarcoma e metástases ${ }^{14}$.

O tratamento do GE depende de fatores como a localização, número de lesões e idade do paciente. Em caso de lesão única e circunscrita, a curetagem com a excisão completa tem sido o procedimento mais adequa$\mathrm{do}^{5,11,12,23,24,30,31}$. Esse procedimento tem sido bastante eficaz em crianças pequenas, pois se evita o uso de radioterapia complementar com suas conhecidas complicações. Em casos de lesões múltiplas ou recorrentes, indica-se quimioterapia, administração de corticoesteróides na lesão ou por via sistêmica ${ }^{5,31}$. As drogas mais usadas no tratamento sistêmico do GE são: vimblastina, vincristina, etoposídeo e metotrexato, na maioria das vezes utilizadas em associação. Outros agentes têm sido pesquisados, como o ácido retinóico, talidomida, interleucina-2 e ciclosporina, porém há necessidade de melhor avaliação para seu uso rotineiro $^{5}$. Radioterapia é alternativa terapêutica, sendo indicada em casos de lesões múltiplas, recorrentes ou inacessíveis ao tratamento cirúrgico, mas deve ser evitada em crianças com idade inferior a dois anos ${ }^{16,31}$. Nossos pacientes foram submetidos a exérese total da lesão, pois apresentavam lesão única e localizada superficialmente. Os resultados foram excelentes.

A doença apresenta péssimo prognóstico quando é multifocal, há envolvimento do sistema nervoso central e presença de disfunção pulmonar, hepática ou hematopoiética ${ }^{8,21,27}$.

\section{Referências}

1. ALESSI DM, MACERI D: Histiocytosis $X$ of the head and neck in a pediatric population. Arch Otolaryngol Head Neck Surg 118:945-8, 1992.
2. ANGELI SI, HOFFMAN HT, ALCADE J, SMITH RJH: Langerhans' cell histiocytosis of the head and neck in children. Ann Otol Rhinol Laryngol 104:173-80, 1995.

3. ANGELI SI, LUXFORD WM, LO WWM: Magnetic resonance imaging in the evaluation of Langerhans' cell histiocytosis of the temporal bone: case report. Otolaryngol Head Neck Surg 114:120-4, 1996.

4. ARCECI RJ, HOPKINS J: New treatment approaches for patients with LCH: Histiocyte Society News, 2002.

5. ARSENI C, DÃNÃILÃ L, CONSTANTINESCU AI: Cranial eosinophilic granuloma. Neurochirurgia 20:189-99, 1977.

6. BELTRAN J, APARISI F, BONMATI LM, ROSENBERG ZS, PRESENT D , STEINER GC: Eosinophilic granuloma: MRI manifestations. Skeletal Radiol 22:157, 1993.

7. CHO DY, LIAU WR, CHIANG IP: Eosinophilic granuloma with acute epidural hematoma. Pediatr Neurosurg 35:266-9, 2001.

8. DEVANEY KO, FERLITO A, PUTZI JM, RINALDO A: Clinicopathological consultation head and neck Langerhans cell histiocytosis. Ann Otol Rhinol Laryngol 106:526-32, 1997.

9. DINARDO LJ, WETMORE RF: Head and neck manifestations of histiocytosis $X$ in children . Laryngoscope 99:7214, 1989.

10. EMILE J-F, FRAITAG S, ANDRY P, LEBORGNE M, LELLOUCH- TUBIANA A, BROUSSE N: Expression of GM-CSF receptor by Langerhans' cell histiocytosis cells. Virchows Arch 427:125-9, 1995.

11. GOLDBERG R, HAN JS, GANZ E, ROESSMAN U: Computed tomography demonstration of multiple parenchymal central nervous system nodules due to Histiocytosis X. Surg Neurol 27:377-80, 1987.

12. GREENWOODS MS, MARTIN SJ, TOWFIGHI J: Unifocal eosinophilic granuloma of the temporal lobe. Surg Neurol 17:441-4, 1982.

13. HADJIGEORGI C, PARPOUN ASC, ZARMAKOUPIS P, LAFOYIANNI S: Eosinophilic granuloma of the temporal bone: radiological approach in the pediatric patient. Pediatr Radiol 20:546-9, 1990.

14. HERMANS R, FOER BD, SMET HM, LEYSEN J, FEENSTRA L, FOSSION E, BAERT LA: Eosinophilc granuloma of the head and neck: CT and MRI features in three cases. Pediatr Radiol 24:33-6, 1994.

15. KAMIMURA M, KINOSHITA T, ITOH H, YUZAWA $Y$, TAKAHASHI J, OHTSUKA K: Eosinophilic granuloma of the spine: early spontaneous disappearance of tumor detected on magnetic resonance imaging. J Neurosurg 93:312-6, 2000

16. LASSO JM, ERENCHUN RR, BAZÁN A: Eosinophilic granuloma of the orbit producing extensive bony destruction in a 32- month-old male infant. Ann Plastic Surg 44:109-10, 2000.

17. LEE KW, MCLEARY MS, ZUPPAN CW, WON DJ: Langerhans' cell histiocytosis presenting with an intracranial epidural hematoma. Pediatr Radiol 30:3268, 2000.

18. LICHTENSTEIN L: Histiocytosis X: integration of eosinophilic granuloma of bone, "Letterer- Siwe disease" and "Schüller- Christian disease" as related manifestations of a single nosologic entity. Arch Pathol 56:85-102, 1953.

19. MARTINEZ-LAGE JF, POZA M, CARTAGENA J, VOCENTE JP, BIEC F, DE LAS HERAS M: Solitary eosinophilic granuloma of the pediatric skull and spine. The role of surgery. Child's Nerv Syst 7:448-51, 1991.

20. MARTINI A, AIMONI C, TREVISANI M, MARANGONI $P$ : Langerhans' cell histiocytosis: report of a case with temporal localization. Inter J Pediatr Otorhinolaryngol 55:51-6, 2000. 
21. McCAFFREY VT, MCDONALD JT: Histiocytosis $X$ of the ear and temporal bone: review of 22 cases. Laryngocospe 39:1735-42, 1979

22. MILLER C, LLOYD TV, JOHNSON JC, HUNT WE: Eosinophilic granuloma of the base of the skull. J Neurosurg 49:464-6, 1978.

23. MONTINE TJ, HOLLENSEAD SC, ELLIS WG, MARTIN JS, MOFFAT EJ, BURGER PC: Solitary eosinophilic granuloma of the temporal lobe: a case report and longterm follow-up of previously reported cases: Clin Neuropathol 13:225-8, 1994.

24. PAILLAS JE, LAVIEILLE J, GOMEZ A: Granulomes éosinophiles du crane. Forme typique et formes atypiques. Sem Hôp Paris 8:2609-16, 1973.

25. PEREIRA CU, SOUSA MRP, GODINHO AS, LEÃO JDBC: Tumores benignos e lesões pseudotumorais do crânio. Arq Bras Neurocir 20:94-100, 2001

26. RAWLINGS CE, WILKINS RH: Solitary eosinophilic granuloma of the skull. Neurosurgery 15:155-61, 1984.

27. RIERA L, VILASECA I, MORELLO A, GASTON F, CALVO C: Histiocytosis de células de Langerhans de hueso temporal. Acta Otorrinolaring Esp 51:357-60, 2000.
28. RUBÉ J, DE LA PAVA S, PICKREN WJ: Histiocytosis $X$ with involvement of brain. Cancer 20:486-92, 1967.

29. SWEET RM, KORNBLUT AD, HYAMS VJ: Eosinophilic granuloma in the temporal bone. Laryngoscope 89:154552, 1979.

30. TOOHILL RJ, KIDDER TM, EBY LG: Eosinophilic granuloma of the temporal bone. Laryngoscope 6:877- 89, 1973.

31. YETISER S, KARAHATAY S, DEVECI S: Eosinophilic granuloma of the bilateral temporal bone. Inter J Pediatr Otorrinolaryngol 62:169-73, 2002.

Original recebido em novembro de 2003

Aceito para publicação em outubro de 2003

\section{Endereço para correspondência:}

Prof. Dr. Carlos Umberto Pereira

Av. Augusto Maynard, 245/404

CEP 49015-380 - Aracaju, SE

E-mail: umberto@infonet.com.br 